\title{
REGULARITY OF STABLE SOLUTIONS TO QUASILINEAR ELLIPTIC EQUATIONS ON RIEMANNIAN MODELS
}

\author{
Rodrigo G. Clemente and João Marcos do Ó \\ Rural Federal University of Pernambuco, Department of Mathematics \\ 52171-900, Recife, Pernambuco, Brazil; rodrigo.clemente@ufrpe.br \\ Brasília University, Department of Mathematics \\ 70910-900, Brasília, DF, Brazil; jmbo@pq.cnpq.br
}

\begin{abstract}
We investigate the regularity of semi-stable, radially symmetric, and decreasing solutions for a class of quasilinear reaction-diffusion equations in the inhomogeneous context of Riemannian manifolds. We prove uniform boundedness, Lebesgue and Sobolev estimates for this class of solutions for equations involving the $p$-Laplace Beltrami operator and locally Lipschitz non-linearity. We emphasize that our results do not depend on the boundary conditions and the specific form of the non-linearities and metric. Moreover, as an application, we establish regularity of the extremal solutions for equations involving the $p$-Laplace Beltrami operator with zero Dirichlet boundary conditions.
\end{abstract}

\section{Introduction}

Let $(\mathcal{M}, g)$ be a Riemannian model of dimension $N \geq 2$, that is, a manifold $\mathcal{M}$ admitting a pole $\mathcal{O}$ and whose metric $g$ is given, in polar coordinates around $\mathcal{O}$, by

$$
\mathrm{d} s^{2}=\mathrm{d} r^{2}+\psi(r)^{2} \mathrm{~d} \theta^{2} \quad \text { for } r \in(0, R) \text { and } \theta \in \mathbf{S}^{N-1},
$$

where $r$ is by construction the Riemannian distance between the point $P=(r, \theta)$ to the pole $\mathcal{O}, \mathrm{d} \theta^{2}$ is the canonical metric on the unit sphere $\mathbf{S}^{N-1}$ and $\psi$ is a smooth function in $[0, R)$ and positive in $(0, R)$ for some $R \in(0,+\infty]$, and $\psi(0)=\psi^{\prime \prime}(0)=$ 0 and $\psi^{\prime}(0)=1$. As examples we have the important cases of space forms, i.e., the unique complete and simply connected Riemannian manifold of constant sectional curvature $K_{\psi}$ corresponding to the choice of $\psi$ namely,

(i) $\psi(r)=\sinh r, \quad K_{\psi}=-1 \quad$ (hyperbolic space),

(ii) $\psi(r)=r, \quad K_{\psi}=0 \quad$ (Euclidean space),

(iii) $\psi(r)=\sin r, \quad K_{\psi}=1 \quad$ (elliptic space).

Let us denote the geodesic ball of radius $\mathrm{r}$ with center at the pole $\mathcal{O}$ by $\mathcal{B}_{r}$ and $W_{r}^{1, p}\left(\mathcal{B}_{1}\right)$ the elements of the Sobolev space which are radially symmetric with respect to the pole $\mathcal{O}$. For $u \in W_{r}^{1, p}\left(\mathcal{B}_{1}\right)$, let us consider the energy functional

$$
J_{\delta}(u)=\frac{1}{p} \int_{\mathcal{B}_{1} \backslash \overline{\mathcal{B}_{\delta}}}\left|\nabla_{g} u\right|^{p} \mathrm{~d} v_{g}-\int_{\mathcal{B}_{1} \backslash \overline{\mathcal{B}_{\delta}}} F(u) \mathrm{d} v_{g}, \text { where } F(x, t)=\int_{0}^{t} f(x, s) \mathrm{d} s .
$$

https://doi.org/10.5186/aasfm.2019.4448

2010 Mathematics Subject Classification: Primary 35B35, 35D10, 35J62, 35J70, 35J75.

Key words: Nonlinear PDE of elliptic type, $p$-Laplacian, singular non-linearity, semi-stable solutions, extremal solutions, regularity.

Research supported in part by INCTmat/MCT/Brazil, CNPq and CAPES.

Corresponding author: J. M. do Ó. 
Definition 1.1. We say that a decreasing function $u \in W_{r}^{1, p}\left(\mathcal{B}_{1}\right)$ is a radial local minimizer of (1.3) if for any $0<\delta<1$ there exists $\epsilon=\epsilon(\delta)>0$ such that for all radial functions $\phi \in C_{c}^{1}\left(\mathcal{B}_{1} \backslash \overline{\mathcal{B}_{\delta}}\right)$ satisfying $\|\phi\|_{C^{1}} \leq \epsilon$, we have

$$
J_{\delta}(u) \leq J_{\delta}(u+\phi)
$$

Definition 1.2. Let $u \in W_{r}^{1, p}\left(\mathcal{B}_{1}\right)$ be a critical point of (1.3). We say that $u$ is semi-stable if $u_{r}(r)<0$ for all $r \in(0,1)$ and for all radially symmetric function $\xi \in C_{c}^{1}\left(\mathcal{B}_{1} \backslash\{\mathcal{O}\}\right)$ it holds

$$
\int_{\mathcal{B}_{1}}\left[(p-1)|\nabla u|^{p-2}|\nabla \xi|^{2}-f^{\prime}(u) \xi^{2}\right] \mathrm{d} v_{g} \geq 0 .
$$

We note that critical points of the functional $J_{\delta}$ correspond to weak solutions of the singular problem

$$
-\operatorname{div}\left(\left|\nabla_{g} u\right|^{p-2} \nabla_{g} u\right)=f(u) \quad \text { in } \quad \mathcal{B}_{1} \backslash\{\mathcal{O}\} .
$$

In particular, if $u$ is a radial local minimizer of $J_{\delta}$, then $u$ is a semi-stable solution of $(\mathcal{S})$. We are going to focus our analysis on the important case $1<p \leq N$, since for $p>N$, it holds $W^{1, p}\left(\mathcal{B}_{1}\right) \hookrightarrow L^{\infty}\left(\mathcal{B}_{1}\right)$. Precisely, if $N<p \leq+\infty$, from Morrey's inequality we have $\|u\|_{C^{0, \gamma}\left(\mathcal{B}_{1}\right)} \leq C\|u\|_{W^{1, p}\left(\mathcal{B}_{1}\right)}$, where $C$ is a constant which depends on $p$ and $N$.

1.1. Main results and comments. The aim of the paper is twofold. Firstly, to establish a priori estimates for radial semi-stable classical solutions of $(\mathcal{S})$. Precisely, we establish $L^{\infty}, L^{q}$ and $W^{1, q}$ estimates for semi-stable, radially symmetric, and decreasing solutions of $(\mathcal{S})$ without assuming Dirichlet boundary condition or any other kind of boundary conditions. It should be an interesting question to study similar results for non-radial solutions. We stress that our results hold for any locally Lipschitz non-linearity $f(s)$ and metric $g$ satisfying (1.1).

Since the celebrated paper by Gidas, Ni and Nirenberg [24] the question of symmetry in non-linear partial differential equations has been the subject of intensive investigations. In [16], by using a variant of moving planes method, it was established radial symmetry for non-negative solutions of quasilinear elliptic equations defined in geodesic balls of the hyperbolic space $\mathbf{H}^{n}$ with homogeneous Dirichlet boundary condition.

Theorem 1.1. For any $f(s)$ locally Lipschitz function and $u$ semi-stable solution of $(\mathcal{S})$, it holds that:

(a) If $N<p+4 p /(p-1)$, then $u \in L^{\infty}\left(\mathcal{B}_{1}\right)$ and

$$
\|u\|_{L^{\infty}\left(\mathcal{B}_{1}\right)} \leq C_{N, p, \alpha, \psi}\|u\|_{L^{p}\left(\mathcal{B}_{1}\right)} .
$$

(b) If $N \geq p+4 p /(p-1)$, then $u \in L^{q}\left(\mathcal{B}_{1}\right)$ and

$$
\|u\|_{L^{q}\left(\mathcal{B}_{1}\right)} \leq C_{N, \psi, p, q}\|u\|_{L^{p}\left(\mathcal{B}_{1}\right)} \quad \text { for any } \quad q<q_{0}:=\frac{N p}{N-p-2-2 \sqrt{\frac{N-1}{p-1}}} .
$$

Moreover, $u \in W^{1, q}\left(\mathcal{B}_{1}\right)$ and

$$
\|u\|_{W^{1, q}\left(\mathcal{B}_{1}\right)} \leq C_{N, \psi, p, q}\|u\|_{L^{p}\left(\mathcal{B}_{1}\right)} \quad \text { for any } \quad q<q_{1}:=\frac{N p}{N-2-2 \sqrt{\frac{N-1}{p-1}}} .
$$


Remark 1.1. For our argument in the proof of Theorem 1.1 it was crucial the following key estimate for semi-stable solutions of $(\mathcal{S})$

$$
\int_{0}^{\delta}\left|u_{r}\right|^{p} \psi^{N-1-2 \alpha} \mathrm{d} r \leq C_{N, p, \alpha, \psi}\|u\|_{L^{p}\left(\mathcal{B}_{1}\right)}^{p},
$$

where $\psi$ is the polar decomposition of $\mathrm{d} s^{2}$ given in (1.1) (see Proposition 3.1 below). We have proved this estimate by using the radial symmetry of the solution $u$ and by choosing an appropriated test function in the semi-stability inequality (1.4),

Remark 1.2. From Theorem $1.1(a)$, one can see that Problem $(\mathcal{S})$ does not have any singular solution.

Remark 1.3. Note that $q_{0}>p^{*}=(N p) /(N-p)$ (the critical Sobolev exponent) and $q_{1}>p$. Under the hypotheses of Theorem 1.1 if $N \geq p+4 p /(p-1)$ then $u$ belongs to $L^{q}\left(\mathcal{B}_{1}\right)$ for all $q<q_{0}$. Since $q_{0}$ is greater than the critical Sobolev exponent, from Theorem 1.1 (ii) we conclude that semi-stable radially symmetric and decreasing weak solutions of $(\mathcal{S})$ have a better regularity than the one expected by using the classical Sobolev embedding. Moreover, we established better regularity than $W^{1, p}$ for semi-stable solutions to Problem $(\mathcal{S})$, since our estimates shows an improvement in the Sobolev space $W^{1, q}$ for $q<q_{1}$.

Our second purpose of this work is to apply the elliptic estimates obtained in Theorem 1.1 to prove regularity results for the following class of quasilinear elliptic problems

$$
\begin{cases}-\operatorname{div}\left(\left|\nabla_{g} u\right|^{p-2} \nabla_{g} u\right)=\lambda h(u) & \text { in } \mathcal{B}_{1}, \\ u>0 & \text { in } \mathcal{B}_{1}, \\ u=0 & \text { on } \partial \mathcal{B}_{1}\end{cases}
$$

where $\lambda$ is a positive parameter and $h(s)$ is an increasing $C^{1}$-function such that $h(0)>0$ and

$$
\lim _{t \rightarrow+\infty} \frac{h(t)}{t^{p-1}}=+\infty
$$

The study of this class of problems with various boundaries conditions has received considerable attention in recent years under the influence of the pioneering works of Gelfand [22], Joseph and Lundgren [28], Keener and Keller [31], Crandall and Rabinowitz [12], Mignot and Puel [37]. First, we would like to mention the progress involving Laplacian

$$
\begin{cases}-\Delta u=\lambda h(u) & \text { in } \Omega \\ u=0 & \text { on } \partial \Omega\end{cases}
$$

where $\Omega$ is a bounded domain of $\mathbf{R}^{N}$. Non-linear elliptic problems like (1.6) appear naturally in several physical phenomena, just to mention some applications, it arises in the theory of non-linear diffusion generated by non-linear sources [26, 27, 29], thermal ignition of a chemically active mixture of gases [22], membrane buckling [7] and gravitation equilibrium [11]. We refer the reader to [13, 17, 18] for a recent survey on this subject.

In recent years, regularity issues about this class of singular elliptic problems have been the focus of an active research area. The parameter $\lambda$ measure the nondimensional strength of the non-linearity. It is well known that if $h$ is super-linear, 
there exists $\lambda^{*} \in(0,+\infty)$ such that if $\lambda \in\left(0, \lambda^{*}\right)$, then problem (1.6) admits a semistable solution $u_{\lambda}$ and if $\lambda>\lambda^{*}$, then problem (1.6) admits no regular solution. This allows one to define the extremal solution $u^{*}:=\lim _{\lambda \gamma^{*}} u_{\lambda}$, which is a weak solution of (1.6). In [40], G. Nedev proved regularity results for extremal solutions of (1.6) in dimensions 2 and 3 and $L^{q}$ estimates for every $q<N /(N-4)$ when $N \geq 4$ just assuming that $h(s)$ is a positive convex function with $h(0)>0$ and $h^{\prime}(0) \geq 0$. For a related problem still in the Euclidean case see [3], where X. Cabré assuming $h(s)$ to be a $C^{1}$ non-decreasing super-linear non-linearity with $h(0)>0$, proved boundedness of the extremal solution for Problem (1.6) in dimension $N \leq 4$. In dimension 2 the domain $\Omega$ can be general but, in contrast with Nedev's result, in dimensions 3 and 4 the domain is assumed to be convex. After that, Cabré and Sanchón [6] completed the analysis in [3] when they proved that if $N \geq 5$ and $\Omega$ is a convex bounded domain of Euclidean space $\mathbf{R}^{N}$ then the extremal solution belongs to $L^{\frac{2 N}{N-4}}$.

Recently, there has been growing interest on singular elliptic partial differential equations on Riemannian manifolds. The problem involving the Laplace-Beltrami operator

$$
\begin{cases}-\Delta_{g} u=\lambda h(u) & \text { in } \Omega, \\ u=0 & \text { on } \partial \Omega\end{cases}
$$

where $\Omega$ is a bounded domain was studied recently by Castorina and Sanchón in [8] for the inhomogeneous context. They proved qualitative properties for semi-stable solutions and they established $L^{\infty}, L^{q}$ and $W^{1, q}$ estimates which do not depends on the non-linearity $h(s)$. Furthermore, the authors obtained regularity results for the extremal solution for exponential and power non-linearities. A similar setting has been considered by Berchio, Ferrero and Grillo [2] in order to study uniqueness and qualitative properties of radial entire solutions of the Lane-Emden-Fowler equation $-\Delta u=|u|^{m-1} u$ with $m>1$ on certain classes of Cartan-Hadamard manifolds where the so-called Joseph-Lundgren exponent is involved in the stability of solutions. The existence of a stable solution to the semi-linear equation $-\Delta_{g} u=f(u)$ on a complete, non-compact, boundaryless Riemannian manifold with non-negative Ricci curvature and $f \in C^{1}$ was studied by Farina, Mari and Valdinoci [19]. They classify both the solution and the manifold and also discuss the classification of monotone solutions with respect to the direction of some Killing vector field, in the spirit of a conjecture of De Giorgi. In [39], Morabito investigated the existence and uniqueness of positive radial solutions of the problem

$$
\begin{cases}\Delta_{g} u+\lambda u+u^{p}=0 & \text { in } \mathcal{A} \\ u=0 & \text { on } \mathcal{A}\end{cases}
$$

when $\lambda<0, \mathcal{A}$ is an annular domain in a Riemannian manifold of dimension $N$ endowed with the metric $\mathrm{d} s^{2}=\mathrm{d} r^{2}+S^{2}(r) \mathrm{d} \theta^{2}$ under suitable assumptions on the function $S^{2}(r)$. He also show that there exist positive non-radial solutions arising by bifurcation from the radial solution, where $\lambda$ and $p$ are the bifurcation parameters.

Many non-linear problems in physics and mechanics are formulated in equations that contain the $p$-Laplacian, for example on non-Newtonian fluids, glaceology and non-linear elasticity (see [14]). For some problems of non-linear partial differential equations on Riemannian manifold we refer to [25, 30, 23]. Gelfand type problems 
involving the $p$-Laplacian in the homogeneous case of the form

$$
\begin{cases}-\Delta_{p} u=\lambda h(u) & \text { in } \Omega \\ u>0 & \text { in } \Omega \\ u=0 & \text { on } \partial \Omega\end{cases}
$$

was studied by García-Azorero, Peral and Puel [20, 21] where $\Omega$ is a smooth bounded domain of $\mathbf{R}^{N}$. They proved that for every $p>1$ and $h(s)=e^{s}$, the extremal solution $u^{*}$ is an energy solution for every dimension and that it is bounded in some range of dimensions. For a more general non-linearity, Cabré and Sanchón [5] proved that every semi-stable solution is bounded for a explicit exponent which is optimal for the boundedness of semi-stable solutions and, in particular, it is bigger than the critical Sobolev exponent $p^{*}-1$. For general $h(s)$ and $p>1$ the interested reader can see $[4,10,41,43]$ for more regularity results about the extremal solution. In [4], Cabré, Capella and Sanchón treated the delicate issue about regularity of extremal solutions $u^{*}$ of (1.7) at $\lambda=\lambda^{*}$ when $\Omega$ is the unit ball of $\mathbf{R}^{N}$. Among other results, they established pointwise, $L^{q}$ and $W^{1, q}$ estimates which are optimal and do not depend on the non-linearity $h(s)$.

Furthermore, Castorina and Sanchón [9] obtain a priori estimates for semi-stable solutions of the reaction-diffusion problem $-\Delta_{p} u=h(u)$ in $\Omega$ while the reaction term is driven by any positive $C^{1}$ non-linearity $h$ and, as a main tool, they develop Morrey-type and Sobolev-type inequalities that involve the functional

$$
I_{p, q}(v ; \Omega)=\left(\int_{\Omega}\left[\left(\left.\frac{1}{p^{\prime}}\left|\nabla_{T, v}\right| \nabla v\right|^{p / q} \mid\right)^{q}+\left|H_{v}\right|^{q}|\nabla v|^{p}\right] d x\right)^{1 / p}, \quad p, q \geq 1,
$$

where $v \in C_{0}^{\infty}(\bar{\Omega})$. In (1.8), $H_{v}(x)$ denotes the mean curvature at $x$ of the hypersurface $\{y \in \Omega:|v(y)|=|v(x)|\}$ and $\nabla_{T, v}$ is the tangential gradient along a level set of $|v|$. In addition to being of independent interest, these geometric inequalities are used, together with judicious choice of test functions in the semi-stability condition, to obtain their a priori estimates for semi-stable solutions.

In this paper we investigate similar results in the inhomogeneous context of a Riemannian manifold. We use some ideas of [5], comparison principle for $-\Delta_{p}$ (because it is uniformly elliptic) and the positivity of the first eigenvalue (as well the corresponding eigenfunction) of $-\Delta_{p}$ on $\Omega$ (cf. $[1,36,38]$ ). We point out that the regularity results achieved in this paper represent a geometrical extension of the ones obtained for the Euclidean case in [4].

Before we state our main result on the regularity of semi-stable solutions for $\left(\mathcal{P}_{\lambda}\right)$, let us introduce some basic definitions. We say that $u \in W_{0}^{1, p}\left(\mathcal{B}_{1}\right)$ is a weak solution of $\left(\mathcal{P}_{\lambda}\right)$ if $h(u) \in L^{1}\left(\mathcal{B}_{1}\right)$ and

$$
\int_{\mathcal{B}_{1}}|\nabla u|^{p-2} \nabla u \cdot \nabla \phi \mathrm{d} v_{g}=\int_{\mathcal{B}_{1}} h(u) \phi \mathrm{d} v_{g},
$$

for all $\phi \in C_{0}^{\infty}\left(\mathcal{B}_{1}\right)$. Furthermore, by minimal solution we mean smaller than any other super-solution of the Problem $\left(\mathcal{P}_{\lambda}\right)$ and regular solution means that a weak solution $u$ of $\left(\mathcal{P}_{\lambda}\right)$ is $C^{1, \beta}\left(\mathcal{B}_{1}\right)$.

Let us state the existence and basic properties of touchdown parameter.

Theorem 1.2. There exist $\lambda^{*} \in(0, \infty)$ such that

(i) for $0<\lambda<\lambda^{*}$, the problem $\left(\mathcal{P}_{\lambda}\right)$ has a regular minimal solution $u_{\lambda}$,

(ii) for $\lambda>\lambda^{*},\left(\mathcal{P}_{\lambda}\right)$ admits no weak solution, 
(iii) the map $\lambda \rightarrow u_{\lambda}$ is increasing.

As a consequence of Theorem 1.2, the increasing limit

$$
u^{*}=\lim _{\lambda \nearrow \lambda^{*}} u_{\lambda}
$$

is well defined by the point-wise increasing property. If $u^{*}$ is a weak solution of $\left(\mathcal{P}_{\lambda^{*}}\right)$, then $u^{*}$ is called the extremal solution. Since the extremal solutions can be obtained as the limit of classical minimal solutions, our next result is useful in order to prove that $u^{*}$ has the same regularity properties as the ones stated in Theorem 1.1. For this, we need to bound $u^{p-1}$ and $h(u)$ in $L^{1}\left(\mathcal{B}_{1}\right)$ uniformly in $\lambda$. This is possible because we have the growth condition $\left(H_{1}\right)$ on $h(s)$ and the radially decreasing property of the minimal solutions $u_{\lambda}$. Let us now state precisely our results for $\left(\mathcal{P}_{\lambda}\right)$.

Theorem 1.3. Suppose that $N<p+4 p /(p-1)$ and let $h(s)$ be a positive and increasing $C^{1}$-function satisfying $\left(H_{1}\right)$. Then $u^{*}$ is a semi-stable solution of $\left(P_{\lambda^{*}}\right)$ and $u^{*} \in L^{\infty}\left(\mathcal{B}_{1}\right)$.

1.2. Outline. In the next section we bring basic facts about the $p$-Laplace Beltrami operator which will be used through the paper. In Section 2 we prove existence of extremal parameter $\lambda^{*}$ and minimal solutions of $\left(\mathcal{P}_{\lambda}\right)$ for $0<\lambda<\lambda^{*}$. In Section 3 we prove that key-estimate (1.5) by using a suitable choice of test functions under the semi-stability property. In Section 4 we use (1.5) to prove our main theorem about regularity for radially symmetric and decreasing semi-stable solutions of Problem $(\mathcal{S})$ and apply this results for the study of the regularity of extremal solutions of $\left(\mathcal{P}_{\lambda}\right)$.

\section{Proof of Theorem 1.2}

Our first proposition establishes the analog of the classical results for $\left(\mathcal{P}_{\lambda}\right)$ in the Euclidean case. Using some ideas coming from Cabré and Sanchón [5] and Luo, Ye and Zhou [35] we prove the existence of a critical parameter $\lambda^{*}$ which is related with the resolvability of $\left(\mathcal{P}_{\lambda}\right)$.

Proof of Theorem 1.2. For (i), let $w \in W_{0}^{1, p}\left(\mathcal{B}_{1}\right)$ a weak solution of

$$
-\operatorname{div}\left(\left|\nabla_{g} w\right|^{p-2} \nabla_{g} w\right)=1 \quad \text { in } \mathcal{B}_{1},
$$

that is

$$
\int_{\mathcal{B}_{1}}\left|\nabla_{g} w\right|^{p-2} \nabla_{g} w \cdot \nabla_{g} \phi \mathrm{d} \sigma=\int_{\mathcal{B}_{1}} \phi \mathrm{d} \sigma, \quad \forall \phi \in C_{0}^{\infty}\left(\mathcal{B}_{1}\right)
$$

We can see that $w \in C^{1, \alpha}\left(\overline{\mathcal{B}_{1}}\right)$ by using $C^{1, \alpha}$-regularity results (see $[33,15,42]$ ). By the Maximum principle [1, Theorem 3.3] $w$ is non-negative in $\mathcal{B}_{1}$. It is easy verify that 0 is sub-solution of $\left(\mathcal{P}_{\lambda}\right)$ and if $\lambda \leq \lambda_{0}:=1 / h\left(\max _{\overline{\mathcal{B}_{1}}} w\right)$,

$$
\int_{\mathcal{B}_{1}}\left|\nabla_{g} w\right|^{p-2} \nabla_{g} w \cdot \nabla_{g} \phi \mathrm{d} \sigma=\int_{\mathcal{B}_{1}} \phi \mathrm{d} \sigma \geq \int_{\mathcal{B}_{1}} \lambda h(w) \phi \mathrm{d} \sigma
$$

that is, $w$ is a super-solution of $\left(\mathcal{P}_{\lambda}\right)$. Thus, for any $\lambda \leq \lambda_{0}$, Problem $\left(\mathcal{P}_{\lambda}\right)$ has a weak solution $u \in W_{0}^{1, p}\left(\mathcal{B}_{1}\right)$ given by Sub and Super-solution Method (see [32]) with $0 \leq u \leq w$ in $\overline{\mathcal{B}_{1}}$. This implies that $u \in C^{1, \alpha}\left(\overline{\mathcal{B}_{1}}\right)$. As any regular solution $u$ of $\left(\mathcal{P}_{\lambda}\right)$ is also a super-solution for $\left(P_{\mu}\right)$ if $\mu \in(0, \lambda)$, the set of $\lambda$ for which $\left(\mathcal{P}_{\lambda}\right)$ admits a regular solution is an interval. For $(i i)$ we will show that for $\lambda$ sufficient large, there 
is no regular solution for $\left(\mathcal{P}_{\lambda}\right)$, so $\lambda^{*}<+\infty$. It is well known that for the non-linear eigenvalue problem

$$
\begin{cases}-\operatorname{div}\left(\left|\nabla_{g} v_{1}\right|^{p-2} \nabla_{g} v_{1}\right)=\lambda_{1}\left|v_{1}\right|^{p-2} v_{1} & \text { in } \mathcal{B}_{1}, \\ v_{1}=0 & \text { in } \partial \mathcal{B}_{1},\end{cases}
$$

there exists a smaller positive and simple eigenvalue $\lambda_{1}$ with a positive eigenfunction $v_{1}$ in $\mathcal{B}_{1}$. Now, suppose that $\left(\mathcal{P}_{\lambda}\right)$ admits a regular solution $u$ for $\lambda>\lambda_{1}$. The regularity result in [33] give that $v_{1} \in C^{1, \alpha}\left(\overline{\mathcal{B}_{1}}\right)$. By homogeneity, we can assume that $\left\|v_{1}\right\|_{\infty}<h(0)^{\frac{1}{p-1}}$. Note that

$$
-\operatorname{div}\left(\left|\nabla_{g} v_{1}\right|^{p-2} \nabla_{g} v_{1}\right)=\lambda_{1} v_{1}^{p-1} \leq \lambda_{1} h(0)<\lambda h(u)=-\operatorname{div}\left(\left|\nabla_{g} u\right|^{p-2} \nabla_{g} u\right) .
$$

By the comparison principle [1] we have that $v_{1} \leq u$. Let us to take $v_{2}$ a solution of

$$
\begin{cases}-\operatorname{div}\left(\left|\nabla_{g} v_{2}\right|^{p-2} \nabla_{g} v_{2}\right)=\left(\lambda_{1}+\epsilon\right) v_{1}^{p-1} & \text { in } \mathcal{B}_{1} \\ v_{2}=0 & \text { in } \partial \mathcal{B}_{1}\end{cases}
$$

where $\epsilon$ is a positive constant. For $\lambda>\frac{\lambda_{1}+\epsilon}{h(0)} \max _{\overline{\mathcal{B}_{1}}} u^{p-1}$ we obtain

$-\operatorname{div}\left(\left|\nabla_{g} v_{2}\right|^{p-2} \nabla_{g} v_{2}\right)=\left(\lambda_{1}+\epsilon\right) v_{1}^{p-1} \leq\left(\lambda_{1}+\epsilon\right) u^{p-1} \leq \lambda h(u)=-\operatorname{div}\left(\left|\nabla_{g} u\right|^{p-2} \nabla_{g} u\right)$.

Using the comparison principle again we obtain $v_{1} \leq v_{2} \leq u$. Now, let us define recursively $u_{n}$ as the unique solution of

$$
\begin{cases}-\operatorname{div}\left(\left|\nabla_{g} v_{n}\right|^{p-2} \nabla_{g} v_{n}\right)=\left(\lambda_{1}+\epsilon\right) v_{n-1}^{p-1} & \text { in } \mathcal{B}_{1} \\ v_{n}=0 & \text { in } \partial \mathcal{B}_{1}\end{cases}
$$

By comparison principle we obtain $v_{1} \leq \cdots \leq v_{n-1} \leq v_{n} \leq u \in C^{1, \alpha}\left(\overline{\mathcal{B}_{1}}\right)$. This implies that $v_{n} \rightarrow u_{\lambda}$ in $W_{0}^{1, p}\left(\mathcal{B}_{1}\right)$ and consequently $u_{\lambda}$ satisfies

$$
\begin{cases}-\operatorname{div}\left(\left|\nabla_{g} u_{\lambda}\right|^{p-2} \nabla_{g} u_{\lambda}\right)=\left(\lambda_{1}+\epsilon\right) u_{\lambda}^{p-1} & \text { in } \mathcal{B}_{1}, \\ u_{\lambda}=0 & \text { in } \partial \mathcal{B}_{1},\end{cases}
$$

which is impossible since the first eigenvalue of $p$-Laplace Beltrami operator is isolated (see $[44,34])$. Define the critical threshold $\lambda^{*}$ as the supremum of $\lambda>0$ for which $\left(\mathcal{P}_{\lambda}\right)$ admits a regular solution. Thus we have that $\lambda^{*}<+\infty$. Note that, by the construction above, $u_{\lambda}$ is independent of the choice of the super-solution. Since any regular solution of $\left(\mathcal{P}_{\lambda}\right)$ is also a super-solution of $\left(\mathcal{P}_{\lambda}\right)$, we can conclude that $u_{\lambda}$ is a regular minimal solution of $\left(\mathcal{P}_{\lambda}\right)$. In order to check (iii), let $\lambda \leq \mu$. Thus, $u_{\mu}$ is a super-solution of $\left(\mathcal{P}_{\lambda}\right)$, which implies that $u_{\lambda} \leq u_{\mu}$, that is, the map $\lambda \rightarrow u_{\lambda}$ is increasing.

\section{A priori estimates}

In this section we prove the principal estimate (1.5), which as we already mention it is the crucial in our argument to obtain the regularity of the semi-stable solutions in Theorem 1.1. The main idea is to apply an appropriate test function in the stability inequality (see Proposition 3.1). The radial form of $(\mathcal{S})$ can be written as follows

$$
-(p-1)\left|u_{r}\right|^{p-2} u_{r r}-\frac{(N-1) \psi^{\prime}}{\psi}\left|u_{r}\right|^{p-2} u_{r}=f(u) \quad \text { with } r \in(0,1) .
$$

Let us discuss a few preliminary estimates which will be used in our argument. In the next Lemma we prove that the second variation of energy associated to $(\mathcal{S})$ is independent of the non-linearity $f(s)$. 
Lemma 3.1. Let $u \in W_{r}^{1, p}\left(\mathcal{B}_{1}\right)$ be a semi-stable solution of $(\mathcal{S})$ satisfying $u_{r}(r)<0$ for all $r \in(0,1)$. Then, for all radially symmetric function $\eta \in C_{c}^{1}\left(\mathcal{B}_{1} \backslash\{\mathcal{O}\}\right)$ it holds

$$
\int_{\mathcal{B}_{1}}\left|u_{r}\right|^{p}\left[(p-1)\left|\eta_{r}\right|^{2}+\frac{\partial}{\partial r}\left(\frac{(N-1) \psi^{\prime}}{\psi}\right) \eta^{2}\right] \mathrm{d} v_{g} \geq 0 .
$$

Proof. We start considering $\eta \in C_{c}^{1}\left(\mathcal{B}_{1} \backslash \mathcal{O}\right)$ be a radial function with compact support in $\mathcal{B}_{1} \backslash \mathcal{O}$ and choosing $\xi=u_{r} \eta$ as test function in (1.4) there holds

$$
\begin{aligned}
0 & \leq \int_{\mathcal{B}_{1}}(p-1)\left|u_{r}\right|^{p-2}\left|\nabla_{g}\left(u_{r} \eta\right)\right|^{2}-f^{\prime}(u)\left|u_{r}\right|^{2} \eta^{2} \mathrm{~d} v_{g} \\
& =\int_{\mathcal{B}_{1}}(p-1)\left|u_{r}\right|^{p}\left|\nabla_{g} \eta\right|^{2}+(p-1)\left|u_{r}\right|^{p-2} \nabla_{g}\left(\eta^{2} u_{r}\right) \nabla_{g}\left(u_{r}\right)-f^{\prime}(u)\left|u_{r}\right|^{2} \eta^{2} \mathrm{~d} v_{g} .
\end{aligned}
$$

On the other hand, multiplying (3.1) by $\left(\eta^{2} u_{r} \psi^{N-1}\right)_{r}$, integrating and using integration by parts we are able to compute

$$
\begin{aligned}
0 & =\int_{0}^{1}(p-1)\left|u_{r}\right|^{p-2} u_{r r}\left(\eta^{2} u_{r} \psi^{N-1}\right)_{r}+\left[\frac{(N-1) \psi^{\prime}}{\psi}\left|u_{r}\right|^{p-2} u_{r}+g(u)\right]\left(\eta^{2} u_{r} \psi^{N-1}\right)_{r} \mathrm{~d} v_{g} \\
& =\int_{0}^{1}(p-1)\left|u_{r}\right|^{p-2} u_{r r}\left(\eta^{2} u_{r} \psi^{N-1}\right)_{r}-\left[\frac{(N-1) \psi^{\prime}}{\psi}\left|u_{r}\right|^{p-2} u_{r}+g(u)\right]_{r} \eta^{2} u_{r} \psi^{N-1} \mathrm{~d} v_{g},
\end{aligned}
$$

which together with $\partial_{r}\left(\left|u_{r}\right|^{p-2} u_{r}\right)=(p-1)\left|u_{r}\right|^{p-2} u_{r r}$ implies

$$
\begin{aligned}
0= & \int_{0}^{1}(p-1)\left|u_{r}\right|^{p-2} u_{r r} \partial_{r}\left(\eta^{2} u_{r} \psi^{N-1}\right) \mathrm{d} r-\int_{0}^{1} \partial_{r}\left(\frac{(N-1) \psi^{\prime}}{\psi}\right)\left|u_{r}\right|^{p-2} u_{r} \eta^{2} u_{r} \psi^{N-1} \mathrm{~d} r \\
& -\int_{0}^{1} \frac{(N-1) \psi^{\prime}}{\psi}(p-1)\left|u_{r}\right|^{p-2} u_{r r} \eta^{2} u_{r} \psi^{N-1} \mathrm{~d} r-\int_{0}^{1} f^{\prime}(u) u_{r} \eta^{2} u_{r} \psi^{N-1} .
\end{aligned}
$$

Thus

$$
\begin{aligned}
0= & \int_{0}^{1}(p-1)\left|u_{r}\right|^{p-2} u_{r r} \partial_{r}\left(\eta^{2} u_{r}\right) \psi^{N-1} \mathrm{~d} r \\
& -\int_{0}^{1} \partial_{r}\left(\frac{(N-1) \psi^{\prime}}{\psi}\right)\left|u_{r}\right|^{p-2} u_{r}^{2} \eta^{2} \psi^{N-1} \mathrm{~d} r-\int_{0}^{1} f^{\prime}(u) \eta^{2} u_{r}^{2} \psi^{N-1} \mathrm{~d} r
\end{aligned}
$$

which yields

$$
\begin{aligned}
\int_{\mathcal{B}_{1}} \partial_{r}\left(\frac{(N-1) \psi^{\prime}}{\psi}\right)\left|u_{r}\right|^{p} \eta^{2} \mathrm{~d} v_{g}= & \int_{\mathcal{B}_{1}}(p-1)\left|u_{r}\right|^{p-2} u_{r r} \partial_{r}\left(\eta^{2} u_{r}\right) \mathrm{d} v_{g} \\
& -\int_{\mathcal{B}_{1}} f^{\prime}(u) \eta^{2} u_{r}^{2} \mathrm{~d} v_{g} .
\end{aligned}
$$

Using (3.2) and (3.3) we have

$$
\begin{aligned}
0 & \leq \int_{\mathcal{B}_{1}}(p-1)\left|u_{r}\right|^{p-2} u_{r}^{2}|\nabla \eta|^{2}+\int_{\mathcal{B}_{1}}(p-1)\left|u_{r}\right|^{p-2} u_{r r} \nabla\left(\eta^{2} u_{r}\right) \nabla u_{r}-f^{\prime}(u) u_{r}^{2} \eta^{2} \mathrm{~d} v_{g} \\
& =\int_{\mathcal{B}_{1}}(p-1)\left|u_{r}\right|^{p}|\nabla \eta|^{2}+\int_{\mathcal{B}_{1}} \frac{\partial}{\partial r}\left(\frac{(N-1) \psi^{\prime}}{\psi}\right)\left|u_{r}\right|^{p} \eta^{2} \mathrm{~d} v_{g} \\
& =\int_{\mathcal{B}_{1}}\left|u_{r}\right|^{p}\left[(p-1)\left|\eta_{r}\right|^{2}+\frac{\partial}{\partial r}\left(\frac{(N-1) \psi^{\prime}}{\psi} \eta^{2}\right)\right] \mathrm{d} v_{g},
\end{aligned}
$$

which is the desired conclusion. 
We obtain $L^{p}$-estimates for the radial derivative of semi-stable solutions of $(\mathcal{S})$ with the help of Lemma 3.1. For that we consider a suitable class of test functions to analyze the inhomogeneous context of a Riemannian manifold assuming that $p \leq N$ and $1 \leq \alpha<1+\sqrt{(N-1) /(p-1)}$. To be more precise,

Proposition 3.1. Let $u \in W_{r}^{1, p}\left(\mathcal{B}_{1}\right)$ be a semi-stable solution in $\mathcal{B}_{1} \backslash \mathcal{O}$ of $(\mathcal{S})$ satisfying $u_{r}(r)<0$ for $r \in(0,1)$ and $\delta=\delta(\psi) \in(0,1 / 2)$ such that $\psi^{\prime}>0$ in $[0, \delta]$. Then

$$
\int_{0}^{\delta}\left|u_{r}\right|^{p} \psi^{N-1-2 \alpha} \mathrm{d} r \leq C_{N, p, \alpha, \psi}\|u\|_{L^{p}\left(\mathcal{B}_{1}\right)}^{p}
$$

for every $1 \leq \alpha<1+\sqrt{(N-1) /(p-1)}$, where $C_{N, p, \alpha, \psi}$ is a constant depending only on $N, p, \alpha$ and $\psi$.

Proof. Using the semi-stability condition of $u$ and applying Lemma 3.1 with $\psi \eta$ as test function we obtain

$$
(N-1) \int_{\mathcal{B}_{1}}\left[-\psi^{\prime \prime} \psi+\left(\psi^{\prime}\right)^{2}\right]\left|u_{r}\right|^{p} \eta^{2} \mathrm{~d} v_{g} \leq(p-1) \int_{\Omega}\left|u_{r}\right|^{p}\left|(\psi \eta)_{r}\right|^{2} \mathrm{~d} v_{g}
$$

Now, take $\alpha$ satisfying $1 \leq \alpha<1+\sqrt{(N-1) /(p-1)}, \epsilon \in(0,1)$ sufficiently small and

$$
\eta_{\epsilon}(r)= \begin{cases}\psi^{-\alpha}(\epsilon)-\psi^{-\alpha}(\delta) & \text { for } 0 \leq r \leq \epsilon \\ \psi^{-\alpha}(r)-\psi^{-\alpha}(\delta) & \text { for } \epsilon<r \leq \delta \\ 0 & \text { for } \delta<r \leq 1\end{cases}
$$

a Lipschitz function which vanishes on $\partial \mathcal{B}_{1}$. Choosing $\eta=\eta_{\epsilon}$ in the inequality (3.4) we have

$$
\begin{aligned}
& (N-1)\left(\int_{0}^{\epsilon}\left[-\psi^{\prime \prime} \psi+\left(\psi^{\prime}\right)^{2}\right] \eta_{\epsilon}^{2}\left|u_{r}\right|^{p} \psi^{N-1} \mathrm{~d} r+\int_{\epsilon}^{\delta}\left[-\psi^{\prime \prime} \psi+\left(\psi^{\prime}\right)^{2}\right] \eta_{\epsilon}^{2}\left|u_{r}\right|^{p} \psi^{N-1} \mathrm{~d} r\right) \\
& \leq(p-1)\left(\int_{\epsilon}^{\delta}\left[(1-\alpha) \psi^{-\alpha}-\psi^{-\alpha}(\delta)\right]^{2}\left|u_{r}\right|^{p}\left(\psi^{\prime}\right)^{2} \psi^{N-1} \mathrm{~d} r+\int_{0}^{\epsilon} \eta_{\epsilon}^{2}\left|u_{r}\right|^{p}\left(\psi^{\prime}\right)^{2} \psi^{N-1} \mathrm{~d} r\right)
\end{aligned}
$$

which can be written as

$$
\begin{aligned}
& (N-p) \int_{0}^{\epsilon} \eta_{\epsilon}^{2}\left|u_{r}\right|^{p}\left(\psi^{\prime}\right)^{2} \psi^{N-1} \mathrm{~d} r+(N-1) \int_{\epsilon}^{\delta}\left(\psi^{\prime}\right)^{2} \eta_{\epsilon}^{2}\left|u_{r}\right|^{p} \psi^{N-1} \mathrm{~d} r \\
& \leq(p-1) \int_{\epsilon}^{\delta}\left[(1-\alpha) \psi^{-\alpha}-\psi^{-\alpha}(\delta)\right]^{2}\left|u_{r}\right|^{p}\left(\psi^{\prime}\right)^{2} \psi^{N-1} \mathrm{~d} r \\
& \quad+(N-1) \int_{0}^{\delta} \psi^{\prime \prime} \psi\left|u_{r}\right|^{p} \eta_{\epsilon}^{2} \psi^{N-1} \mathrm{~d} r
\end{aligned}
$$

Since $(N-p) \eta_{\epsilon}^{2}\left|u_{r}\right|^{p}\left(\psi^{\prime}\right)^{2} \psi^{N-1} \mathrm{~d} r \geq 0$ we obtain

$$
\begin{aligned}
(N-1) \int_{\epsilon}^{\delta}\left|u_{r}\right|^{p}\left(\psi^{\prime}\right)^{2} \eta_{\epsilon}^{2} \psi^{N-1} \mathrm{~d} r \leq & (p-1) \int_{\epsilon}^{\delta}\left|u_{r}\right|^{p}\left((1-\alpha) \psi^{-\alpha}-\psi^{-\alpha}\right)^{2}\left(\psi^{\prime}\right)^{2} \psi^{N-1} \mathrm{~d} r \\
& +(N-1) \int_{0}^{\delta} \psi^{\prime \prime} \psi\left|u_{r}\right|^{p} \eta_{\epsilon}^{2} \psi^{N-1} \mathrm{~d} r
\end{aligned}
$$

Throughout the proof, $\tilde{C}_{n, p, \alpha}$ (respectively $\tilde{C}_{n, p, \alpha, \psi}$ ) denote different positive constants depending only on $n, p$ and $\alpha$ (respectively on $n, p, \alpha, \psi)$. Rewritten the above 
equation follows

$$
\begin{aligned}
& \int_{\epsilon}^{\delta}\left(\psi^{\prime}\right)^{2}\left|u_{r}\right|^{p} \psi^{-2 \alpha} \psi^{N-1} \mathrm{~d} r \leq \tilde{C}_{n, p, \alpha}\left\{\int_{\epsilon}^{\delta}\left(\psi^{\prime}\right)^{2}\left|u_{r}\right|^{p} \psi^{-2 \alpha}(\delta) \psi^{N-1} \mathrm{~d} r\right. \\
& \left.+\int_{0}^{\delta} \psi^{N-1}\left|u_{r}\right|^{p}\left|\psi^{\prime \prime}\right| \psi\left(\psi^{-2 \alpha}+\psi^{-2 \alpha}(\delta)\right) \mathrm{d} r+\int_{\epsilon}^{\delta}\left(\psi^{\prime}\right)^{2}|u|^{p} \psi^{-\alpha} \psi^{-\alpha}(\delta) \psi^{N-1} \mathrm{~d} r\right\} .
\end{aligned}
$$

Observe that, by assumption, $\inf _{(0, \delta)} \psi^{\prime}$ and $\sup _{(0, \delta)} \psi^{\prime}$ are positive. Now, we can rearrange the terms in the integrals to obtain

$$
\begin{aligned}
\int_{\epsilon}^{\delta}\left|u_{r}\right|^{p} \psi^{-2 \alpha} \psi^{N-1} \mathrm{~d} r \leq & \tilde{C}_{N, p, \alpha} \int_{0}^{\delta} \psi^{N-1}\left|u_{r}\right|^{p}\left|\psi^{\prime \prime}\right| \psi \psi^{-2 \alpha}\left(1+\frac{\psi^{2 \alpha}}{\psi^{2 \alpha}(\delta)}\right) \mathrm{d} r \\
& +\tilde{C}_{N, p, \alpha} \int_{0}^{\delta}\left(\psi^{\prime}\right)^{2}|u|^{p} \psi^{-\alpha} \psi^{-\alpha}(\delta) \psi^{N-1}\left(1+\frac{\psi^{\alpha}}{\psi^{\alpha}(\delta)}\right) \mathrm{d} r \\
\leq & \tilde{C}_{N, p, \alpha, \psi} \int_{0}^{\delta} \psi^{N-1}\left|u_{r}\right|^{p} \psi^{-\alpha}\left\{1+\psi^{1-\alpha}\right\} \mathrm{d} r
\end{aligned}
$$

Taking $\epsilon \rightarrow 0$, follows that

$$
\int_{0}^{\delta}\left|u_{r}\right|^{p} \psi^{-2 \alpha} \psi^{N-1} \mathrm{~d} r \leq \tilde{C}_{N, p, \alpha, \psi} \int_{0}^{\delta} \psi^{N-1}\left|u_{r}\right|^{p} \psi^{-\alpha}\left\{1+\psi^{1-\alpha}\right\} \mathrm{d} r .
$$

If we define

$$
\zeta(t)=\frac{\tilde{C}_{N, p, \alpha, \psi} t^{-\alpha}\left(1+t^{1-\alpha}\right)-\frac{t^{-2 \alpha}}{2}}{t^{\frac{N-1}{p-1}}},
$$

using that $1 \leq p \leq N$ and $\alpha$ satisfying $1 \leq \alpha<1+\sqrt{(N-1) /(p-1)}$, we can check that $\lim _{t \rightarrow+\infty} \zeta(t)=0$ and $\lim _{t \rightarrow 0^{+}} \zeta(t)=-\infty$. Thus, by a compactness argument, $\zeta(t)$ is bounded from above. This implies that there exists $C_{N, p, \alpha, \psi}>0$ such that

$$
\tilde{C}_{N, p, \alpha, \psi} t^{-\alpha}\left(1+t^{1-\alpha}\right) \leq \frac{t^{-2 \alpha}}{2}+C_{N, p, \alpha, \psi} t^{\frac{N-1}{p-1}} \quad \forall t>0
$$

and (3.5) leads to

$$
\int_{0}^{\delta}\left|u_{r}\right|^{p} \psi^{-2 \alpha} \psi^{N-1} \mathrm{~d} r \leq C_{N, p, \alpha, \psi} \int_{0}^{\delta}\left|u_{r}\right|^{p} \psi^{(N-1) p /(p-1)} \mathrm{d} r .
$$

On the other hand, since $u$ is radially decreasing follows that

$$
u^{p}(\delta) \leq C_{N, \psi} \int_{0}^{\delta} u^{p} \psi^{N-1} \mathrm{~d} r \leq C_{N, \psi}\|u\|_{L^{p}\left(\mathcal{B}_{1}\right)}^{p}
$$

and using Mean value theorem for some $\tilde{\delta} \in(\delta, 2 \delta)$ it holds

$$
-u_{r}(\tilde{\delta})=\frac{u(\delta)-u(2 \delta)}{\delta} \leq \frac{u(\delta)}{\delta} .
$$

Thus, integrating (3.1) from $r \in(0, \delta)$ to $\tilde{\delta}$ and using (3.8) we obtain

$$
\begin{aligned}
-\left|u_{r}(r)\right|^{p-2} u_{r}(r) \psi^{N-1}(r) & =-\left|u_{r}(\tilde{\delta})\right|^{p-2} u_{r}(\tilde{\delta}) \psi^{N-1}(\tilde{\delta})-\int_{s}^{\tilde{\delta}} f(u) \psi^{N-1} \\
& \leq \frac{u^{p-1}(\delta)}{\delta^{p-1}} \psi^{N-1}(\tilde{\delta})
\end{aligned}
$$

which together (3.7) implies

$$
\left|u_{r}\right|^{p} \psi^{(N-1) p /(p-1)} \leq \frac{u^{p}(\delta)}{\delta^{p}} \psi^{p(N-1) /(p-1)}(\tilde{\delta}) \leq C_{N, \psi}\|u\|_{L^{p}\left(\mathcal{B}_{1}\right)}^{p} .
$$


Integrating from 0 to $\delta$ to obtain

$$
\int_{0}^{\delta}\left|u_{r}\right|^{p} \psi^{(N-1) p /(p-1)} \mathrm{d} r \leq C_{N, \psi}\|u\|_{L^{p}\left(\mathcal{B}_{1}\right)}^{p}
$$

and going back to (3.6) we conclude that

$$
\int_{0}^{\delta}\left|u_{r}\right|^{p} \psi^{N-1-2 \alpha} \mathrm{d} r \leq C_{N, p, \alpha, \psi}\|u\|_{L^{p}\left(\mathcal{B}_{1}\right)}^{p}
$$

which completes the proof.

\section{Proof of main theorems}

Proof of Theorem 1.1. Let $\delta \in(0,1 / 2)$ as in Proposition 3.1. Since $u$ is radially symmetric and positive we can check that

$$
u(\delta) \leq \int_{0}^{\delta} u \psi^{N-1}, \mathrm{~d} r \leq C_{N, \psi}\|u\|_{L^{1}\left(\mathcal{B}_{1}\right)}
$$

Using Hölder inequality we can estimate

$$
\begin{aligned}
|u(t)| & =\left|u(\delta)-\int_{t}^{\delta} u_{r} \psi^{(N-1-2 \alpha) / p} \psi^{(-N+1+2 \alpha) / p} \mathrm{~d} r\right| \\
& \leq C_{N, \psi}\|u\|_{L^{1}\left(\mathcal{B}_{1}\right)}+\left(\int_{0}^{\delta}\left|u_{r}\right|^{p} \psi^{N-1-2 \alpha}\right)^{\frac{1}{p}}\left(\int_{t}^{\delta} \psi^{(-N+1+2 \alpha) /(p-1)}\right)^{\frac{p-1}{p}} \\
& \leq C_{N, \psi}\|u\|_{L^{1}\left(\mathcal{B}_{1}\right)}+C_{N, p, \alpha, \psi}\|u\|_{L^{p}\left(\mathcal{B}_{1}\right)}\left(\int_{t}^{\delta} \psi^{(-N+1+2 \alpha) /(p-1)}\right)^{\frac{p-1}{p}}
\end{aligned}
$$

(i) In order to prove $L^{\infty}$ estimate, observe that, by monotonicity, for all $\delta \leq t<1$ we have

$$
u^{p}(t) \leq u^{p}(\delta) \leq C_{N, \psi}\|u\|_{L^{p}\left(\mathcal{B}_{1}\right)}^{p} .
$$

Taking $t=0$ in (4.2), we can analyze the integral and check that

$$
\int_{0}^{\delta} \psi^{(2 \alpha-N+1) /(p-1)}<+\infty
$$

when $(2 \alpha-N+1) /(p-1)>-1$, that is, $\alpha>(N-p) / 2$. Thus, for all $0<t<\delta$ we have

$$
|u(t)| \leq C_{N, \psi}\|u\|_{L^{1}\left(\mathcal{B}_{1}\right)}+C_{N, p, \alpha, \psi}\|u\|_{L^{p}\left(\mathcal{B}_{1}\right)},
$$

whenever $\max \{(N-p) / 2,1\}<\alpha<1+\sqrt{(N-1) /(p-1)}$. This occurs if, and only if, $N<p+4 p /(p-1)$. Therefore, the desired $L^{\infty}$ estimate (1.1) holds true.

(ii) On the other hand, since $u$ is decreasing, using (4.1) we have

$$
\left(\int_{\delta}^{1}|u|^{q} \psi^{N-1} \mathrm{~d} t\right)^{\frac{1}{q}} \leq C_{N, \psi, q} u(\delta) \leq C_{N, \psi, q}\|u\|_{L^{1}\left(\mathcal{B}_{1}\right)} .
$$

Taking $t \in(0, \delta)$ and using (4.2) we have

$$
\int_{t}^{\delta}|u|^{q} \psi^{N-1} \mathrm{~d} t \leq C_{N, \psi, q}\|u\|_{L^{p}\left(\mathcal{B}_{1}\right)}^{q} \int_{0}^{\delta}\left[1+\left(\int_{t}^{\delta} \psi^{(-N+1+2 \alpha) /(p-1)}\right)^{\frac{p-1}{p}}\right]^{q} \psi^{N-1} \mathrm{~d} t .
$$


Therefore, if $q<N p /(N-p-2-2 \sqrt{(N-1) /(p-1)})$, choosing suitable $\alpha$ such that

$$
\left(\int_{0}^{\delta}|u|^{q} \psi^{N-1} \mathrm{~d} t\right)^{\frac{1}{q}} \leq C_{N, \psi, q}\|u\|_{L^{p}\left(\mathcal{B}_{1}\right)} .
$$

Taking this last inequality combined with (4.3) we obtain the desired $L^{q}$ estimate. Now, we are looking for $W^{1, q}$ estimate. We use similar idea as can be found in the proof of Theorem 1.2 in [8]. For this, observe that every function $u \in W_{r}^{1, p}\left(\mathcal{B}_{1}\right)$ also belongs to the Sobolev space $W^{1, p}(\delta, 1)$. Thus, we have

$$
\int_{\delta}^{1}\left|u_{r}\right|^{q} \psi^{N-1} \mathrm{~d} r \leq C_{N, q, \psi} \int_{\delta}^{1}\left|u_{r}\right|^{q} \leq C_{N, q, \psi} u^{q}(\delta) \leq C_{N, q, \psi}\|u\|_{L^{q}\left(\mathcal{B}_{1}\right)}^{q} .
$$

Now, using equation (3.1) we have

$$
u_{r r} \leq-\frac{(N-1) \psi^{\prime}}{\psi} u_{r} \quad \text { in }(0,1)
$$

Now, let $\tilde{\delta} \in(\delta, 2 \delta)$ such that (3.8) holds. Integrating the last inequality,

$$
\int_{t}^{\tilde{\delta}} u_{r r} \mathrm{~d} r \leq-(N-1) \int_{t}^{\tilde{\delta}} \frac{\psi^{\prime}}{\psi} u_{r} \mathrm{~d} r
$$

and using (4.1),

$$
\begin{aligned}
-\frac{u_{r}(t)}{N-1} & \leq-\frac{u_{r}(\tilde{\delta})}{N-1}-\int_{t}^{\tilde{\delta}} \frac{\left|\psi^{\prime}\right|}{\psi} u_{r} \mathrm{~d} r \\
& \leq C_{N, \psi}\|u\|_{L^{1}\left(\mathcal{B}_{1}\right)}+\int_{t}^{2 \delta} \frac{\psi^{\prime}}{\psi} \psi^{(-N+1+2 \alpha) / p} \psi^{(N-1-2 \alpha) / p} .
\end{aligned}
$$

Using Hölder inequality and observing that we can use Proposition 3.1 because we can take our $\delta$ sufficiently small, follows

$$
-u_{r}(t) \leq C_{N, p, \psi}\|u\|_{L^{p}\left(\mathcal{B}_{1}\right)}\left(1+\left(\int_{t}^{2 \delta}\left(\frac{\psi^{\prime}}{\psi}\right)^{p^{\prime}} \psi^{p^{\prime}(-N+1+2 \alpha) / p}\right)^{\frac{1}{p^{\prime}}}\right)
$$

for all $\alpha \in[1,1+\sqrt{(N-1) /(p-1)}]$. Thus, for $s \in(0, \delta)$,

$$
\int_{s}^{\delta}\left|u_{r}\right|^{q} \psi^{N-1} \mathrm{~d} r \leq C_{N, p, \psi}\|u\|_{L^{p}\left(\mathcal{B}_{1}\right)}^{q}\left(1+\left(\int_{t}^{2 \delta}\left(\frac{\psi^{\prime}}{\psi}\right)^{p^{\prime}} \psi^{p^{\prime}(-N+1+2 \alpha) / p}\right)^{\frac{1}{p^{\prime}}}\right)^{q}
$$

Now, observe that

$$
\left(\left(\int_{t}^{2 \delta}\left(\psi^{\prime}\right)^{\frac{1}{p^{\prime}}} \psi^{\frac{1}{p-1}(-N+1+2 \alpha-p)}\right)^{\frac{1}{p^{\prime}}}\right)^{q}<C_{N, p, q, \psi}<+\infty
$$

since $q<N p /(N-2-2 \sqrt{(N-1) /(p-1)})$. Thus

$$
\int_{s}^{\delta}\left|u_{r}\right|^{q} \psi^{N-1} \mathrm{~d} r \leq C_{N, p, q, \psi}\|u\|_{L^{p}\left(\mathcal{B}_{1}\right)}^{q} .
$$

Using equations (4.4) and (4.5) we finish the proof. 
Proof of Theorem 1.3. Let $\lambda \in\left(0, \lambda^{*}\right)$. There exists $\rho_{\lambda} \in(1 / 2,1)$ such that mean value property holds, that is,

$$
\frac{\partial u_{\lambda}}{\partial r}\left(\rho_{\lambda}\right)=\frac{u_{\lambda}(1 / 2)-u_{\lambda}(1)}{1 / 2} .
$$

Since $u_{\lambda}$ is decreasing, (see proof of Theorem 1.2), we have

$$
\left[\frac{\partial u_{\lambda}}{\partial r}\left(\rho_{\lambda}\right)\right]^{p-1}=\left[2 u_{\lambda}(1 / 2)\right]^{p-1} \leq C_{N, p, \psi}\left\|u_{\lambda}^{p-1}\right\|_{L^{1}\left(B_{1 / 2}\right)} .
$$

Thus

$$
\left\|\psi^{N-1}\left|\frac{\partial u_{\lambda}}{\partial r}\right|^{p-1}\right\|_{L^{\infty}\left(B_{1 / 2}\right)} \leq C_{N, p, \psi}\left\|u_{\lambda}^{p-1}\right\|_{L^{1}\left(B_{1 / 2}\right)}
$$

follows by monotonicity. By using $\phi(r)=\min \left\{1,(2-4 r)^{+}\right\}$as test function and (4.6) we obtain

$$
\left\|\lambda h\left(u_{\lambda}\right)\right\|_{L^{1}\left(B_{1 / 4}\right)} \leq C_{N, p, \psi} \int_{1 / 4}^{1 / 2} \psi^{N-1}\left|\frac{\partial u_{\lambda}}{\partial r}\right|^{p-1} \mathrm{~d} r \leq C_{N, p, \psi}\left\|u_{\lambda}^{p-1}\right\|_{L^{1}\left(B_{1 / 2}\right)} .
$$

Using the assumption $\left(H_{1}\right)$, given $\delta>0$ we have for any $\lambda \in\left(\lambda^{*} / 2, \lambda^{*}\right)$ and for all $t>0$,

$$
\lambda h(t) \geq \frac{1}{\delta} t^{p-1}-C_{\delta},
$$

where $C_{\delta}$ does not depends on $\lambda$. With this

$$
\left\|u_{\lambda}^{p-1}\right\|_{L^{1}\left(B_{1 / 4}\right)} \leq C_{N, p, \psi} \delta\left\|u_{\lambda}^{p-1}\right\|_{L^{1}\left(B_{1 / 2}\right)}+C_{\delta} .
$$

Since $u_{\lambda}$ is decreasing follows that

$$
\left\|u_{\lambda}^{p-1}\right\|_{L^{1}\left(B_{1 / 2} \backslash \bar{B}_{1 / 4}\right)} \leq C_{N, p, \psi} u_{\lambda}^{p-1}(1 / 4) \leq C_{N, p, \psi}\left\|u_{\lambda}^{p-1}\right\|_{B_{1 / 4}} .
$$

Now, take $\delta$ sufficiently small and combine (4.8) with (4.9) to obtain

$$
\left\|u_{\lambda}^{p-1}\right\|_{L^{1}\left(B_{1 / 4}\right)} \leq C
$$

where $C$ is a constant independent of $\lambda$. Repeating the argument in (4.9) we are able to obtain an estimate uniform in $\lambda$ for $\left\|u_{\lambda}^{p-1}\right\|_{L^{1}\left(B_{1}\right)}$. Using this in (4.7) we obtain a estimate for $\left\|h\left(u_{\lambda}\right)\right\|_{L^{1}\left(B_{1 / 4}\right)}$. Again by monotonicity we can apply the same argument used above to control $\left\|h\left(u_{\lambda}\right)\right\|_{L^{1}\left(B_{1}\right)}$ uniformly in $\lambda$. Thus

$$
\left\|u_{\lambda}^{p-1}\right\|_{L^{1}\left(B_{1}\right)}+\left\|h\left(u_{\lambda}\right)\right\|_{L^{1}\left(B_{1}\right)} \leq C
$$

where $C$ is a constant independent of $\lambda$. Observe that every radial function $u \in$ $W^{1, p}\left(\mathcal{B}_{1}\right)$ also belongs to the Sobolev space $W^{1, p}(\delta, 1)$ in one dimension for a given $\delta \in(0,1)$. Using the Sobolev embedding in one dimension, $u$ becomes a continuous function of $r=\operatorname{dist}(x, \mathcal{O}) \in[\delta, 1]$ and

$$
|u(1)| \leq C_{N, p}\|u\|_{W^{1, p}\left(\mathcal{B}_{1}\right)} .
$$

In view of this estimate, we can assume that $u>0=u(1)$ in $\mathcal{B}_{1}$. Take $\alpha$ satisfying $1 \leq \alpha<1+\sqrt{(N-1) /(p-1)}$ and using Proposition 3.1,

$$
\int_{\mathcal{B}_{1}}\left|u_{r}\right|^{p} \psi^{-2 \alpha} \mathrm{d} v_{g} \leq C_{N, p, \psi} \int_{\mathcal{B}_{1}}\left|u_{r}\right|^{p} \mathrm{~d} x=C_{N, p, \psi} \int_{\mathcal{B}_{r_{0}}}\left|u_{r}\right|^{p} \mathrm{~d} x+C_{N, p, \psi} \int_{\mathcal{B}_{1} \backslash \overline{\mathcal{B}_{r_{0}}}}\left|u_{r}\right|^{p} \mathrm{~d} x .
$$


Now, choose $r_{0}$ such that $2 C_{N, p, \psi} \leq \psi^{-2 \alpha}$ in $r \in\left(0, r_{0}\right)$ to obtain

$$
C_{N, p, \psi} \int_{\mathcal{B}_{r_{0}}}\left|u_{r}\right|^{p} \mathrm{~d} x \leq \frac{1}{2} \int_{\mathcal{B}_{1}} \psi^{-2 \alpha}\left|u_{r}\right|^{p} \mathrm{~d} x
$$

which implies

$$
C_{\psi} \int_{\mathcal{B}_{1}}\left|u_{r}\right|^{p} \mathrm{~d} x \leq \int_{\mathcal{B}_{1}} \psi^{-2 \alpha}\left|u_{r}\right|^{p} \mathrm{~d} x \leq C_{N, p, \psi} \int_{\mathcal{B}_{1} \backslash \overline{\mathcal{B}_{r_{0}}}}\left|u_{r}\right|^{p} \mathrm{~d} x .
$$

Since $u$ is decreasing we have that

$$
u\left(r_{0}\right)^{p-1} \leq C_{N, p}\left\|u^{p-1}\right\|_{L^{1}\left(\mathcal{B}_{r_{0}}\right)} .
$$

Thus,

$$
\begin{aligned}
\int_{\mathcal{B}_{1} \backslash \overline{\mathcal{B}_{0}}}\left|u_{r}\right|^{p} \mathrm{~d} x & =C_{N, \psi} \int_{r_{0}}^{1}\left|u_{r}\right|^{p} \psi^{N-1} \mathrm{~d} r \\
& \leq C_{N, \psi}\left\|\psi^{N-1}\left|u_{r}\right|^{p-1}\right\|_{L^{\infty}\left(\mathcal{B}_{1}\right)} \int_{r_{0}}^{1}-u_{r} \mathrm{~d} r \\
& \leq C_{N, p, \psi}\|h(u)\|_{L^{1}\left(\mathcal{B}_{1}\right)}\left\|u^{p-1}\right\|_{L^{1}\left(\mathcal{B}_{1}\right)}^{\frac{1}{p-1}} .
\end{aligned}
$$

We can conclude that

$$
\int_{\mathcal{B}_{1}}\left|u_{r}\right|^{p} \mathrm{~d} x \leq C_{N, p, \psi} \int_{\mathcal{B}_{1} \backslash \overline{\mathcal{B}_{0}}}\left|u_{r}\right|^{p} \mathrm{~d} x \leq C_{N, p, \psi}\|h(u)\|_{L^{1}\left(\mathcal{B}_{1}\right)}\left\|u^{p-1}\right\|_{L^{1}\left(\mathcal{B}_{1}\right)}^{\frac{1}{p-1}} .
$$

By (4.10) we deduce a bound for $\left\|u_{\lambda}\right\|_{W^{1, p}\left(B_{1}\right)}$. By using the compactness and since $u_{\lambda} \rightarrow u^{*}$ as $\lambda \rightarrow \lambda^{*}$ follows that $u^{*} \in W_{0}^{1, p}\left(B_{1}\right)$. We can pass to the limit and conclude that $u^{*}$ is a weak solution of $\left(\mathcal{P}_{\lambda}\right)$. It is clear that $u^{*}$ is radially symmetric and decreasing. By Fatou's Lemma we obtain that $u^{*}$ is semi-stable. Finally, we can pass to the limit and the regularity statement follows as a consequence of Theorem 1.1.

Corollary 4.1. The extremal solution $u^{*}$ has the same regularity stated in Theorem 1.1.

Proof. The proof follows straightforward by using above estimates and passing to the limit as $\lambda \rightarrow \lambda^{*}$.

\section{References}

[1] Antonini, P., and D. Mugnai, P. Pucci: Quasilinear elliptic inequalities on complete Riemannian manifolds. - J. Math. Pures Appl. 87, 2007, 582-600.

[2] Berchio, E., A. Ferrero, and G. Grillo: Stability and qualitative properties of radial solutions of the Lane-Emden-Fowler equation on Riemannian models. - J. Math. Pures Appl. $102,2014,1-35$.

[3] Cabré, X.: Regularity of minimizers of semilinear elliptic problems up to dimension 4. - Comm. Pure Appl. Math. 63, 2010, 1362-1380.

[4] Cabré, X., A. Capella, and M. Sanchón: Regularity of radial minimizers of reaction equations involving the $p$-Laplacian. - Calc. Var. Partial Differential Equations 34, 2009, 475494.

[5] Cabré, X., and M. Sanchón: Semi-stable and extremal solutions of reaction equations involving the $p$-Laplacian. - Commun. Pure Appl. Anal. 6, 2007, 43-67.

[6] Cabré, X., and M. Sanchón: Geometric-type Sobolev inequalities and applications to the regularity of minimizers. - J. Funct. Anal. 264, 2013, 303-325. 
[7] Callegari, A., E. Reiss, and H. Keller: Membrane buckling. - Comm. Pure Appl. Math. 24, 1971, 499-527.

[8] Castorina, D., and M. Sanchón: Regularity of stable solutions to semilinear elliptic equations on Riemannian models. - Adv. Nonlinear Anal. 4, 2015, 295-309.

[9] CAstorina, D., and M. SAnchón: Regularity of stable solutions of $p$-Laplace equations through geometric Sobolev type inequalities. - J. Eur. Math. Soc. (JEMS) 17, 2015, 2949-2975.

[10] Castorina, D.: Regularity of the extremal solution for singular $p$-Laplace equations. Manuscripta Math. 146, 2015, 519-529.

[11] Chandrasekhar, S.: An introduction to the study of stellar structure. - Dover Publ. Inc., New York, 1985.

[12] Crandall, M., and P. Rabinowitz: Some continuation and variational methods for positive solutions of nonlinear elliptic eigenvalue problems. - Arch. Ration. Mech. Anal. 58, 1975, 207218.

[13] DÁvila, J.: Singular solutions of semi-linear elliptic problems. - In: Handbook of differential equations: stationary partial differential equations. Vol. VI, Handb. Differ. Equ., Elsevier/North-Holland, Amsterdam, 2008, 83-176.

[14] DíAz, J. I.: Nonlinear partial differential equations and free boundaries. Vol. I. Elliptic equations. - Res. Notes Math. 106, Pitman (Advanced Publishing Program), Boston, MA, 1985.

[15] DiBenedetto, E.: $C^{1+\alpha}$ local regularity of weak solutions of degenerate elliptic equations. Nonlinear Anal. 7, 1983, 827-850.

[16] DO Ó, J.M., and R. DA Costa: Symmetry properties for nonnegative solutions of nonuniformly elliptic equations in the hyperbolic space. - J. Math. Anal. Appl. 435, 2016, 17531771.

[17] Dupaigne, L.: Stable solutions of elliptic partial differential equations. - Chapman \& Hall/CRC, Boca Raton, 2011.

[18] Esposito, P., N. Ghoussoub, and N. Guo: Mathematical analysis of partial differential equations modeling electrostatic MEMS. - Courant Lect. Notes Math. 20, Courant Inst. Math. Sci., New York, Amer. Math. Soc., Providence, RI, 2010.

[19] Farina, A., L. MARI, and E. VAldinoci: Splitting theorems, symmetry results and overdetermined problems for Riemannian manifolds. - Comm. Partial Differential Equations 38, 2013, 1818-1862.

[20] García-Azorero, J., and I. Peral: On an Emden-Fowler type equation. - Nonlinear Anal. $18,1992,1085-1097$.

[21] García-Azorero, J., I. Peral, and J. P. Puel: Quasilinear problems with exponential growth in the reaction term. - Nonlinear Anal. 22, 1994, 481-498.

[22] Gelfand, I. M.: Some problems in the theory of quasilinear equations. - Amer. Math. Soc. Transl. 29, 1963, 295-381; Russian original: Uspekhi Mat. Nauk. 14, 1959, 87-158.

[23] Grosjean, J.: p-Laplace operator and diameter of manifolds. - Ann. Global Anal. Geom. 28, $2005,257-270$.

[24] Gidas, B., W. M. Ni, and L. Nirenberg: Symmetry and related properties via the maximum principle. - Comm. Math. Phys. 68, 1979, 209-243.

[25] Holopainen, I.: Asymptotic Dirichlet problem for the $p$-Laplacian on Cartan-Hadamard manifolds. - Proc. Amer. Math. Soc. 130, 2002, 3393-3400.

[26] Joseph, D.: Non-linear heat generation and the stability of the temperature distribution in conducting solids. - Int. J. Heat Mass Transfer 8, 1965, 281-288.

[27] Joseph, D., and D. Cohen: Some positone problems suggested by nonlinear heat generation. - J. Math. Mech. 16, 1967, 1361-1376.

[28] Joseph, D., and T. Lundgren: Quasilinear Dirichlet problem driven by positive sources. Arch. Ration. Mech. Anal. 49, 1972, 241-269. 
[29] Joseph, D., and E. Sparrow: Nonlinear diffusion induced by nonlinear sources. - Quart. Appl. Math. 28, 1970, 327-342.

[30] KAWAI, S., and N. NAKAUChI: The first eigenvalue of the $p$-Laplacian on a compact Riemannian manifold. - Nonlinear Anal. 55, 2003, 33-46.

[31] Keener, J., and H. Keller: Positive solutions of convex nonlinear eigenvalue problems. - J. Differential Equations 16, 1974, 103-125.

[32] KuRA, T.: The weak supersolution-subsolution method for second order quasilinear elliptic equations. - Hiroshima Math. J. 19, 1989, 1-36.

[33] Lieberman, G. M.: Boundary regularity for solutions of degenerate elliptic equations. - Nonlinear Anal. 12, 1988, 1203-1219.

[34] Lindqvist, P.: On the equation $\operatorname{div}\left(|\nabla u|^{p-2} \nabla u\right)+\lambda|u|^{p-2} u=0$. - Proc. Amer. Math. Soc. 109, 1990, 157-164

[35] Luo, X., D. Ye, and F. Zhou: Regularity of the extremal solution for some elliptic problems with singular nonlinearity and advection. - J. Differential Equations 251, 2011, 2082-2099.

[36] MaO, J.: Eigenvalue inequality for the $p$-Laplacian on a Riemannian manifold and estimates for the heat kernel. - J. Math. Pures Appl. 9, 2014, 372-393.

[37] Mignot, F., and J.-P. Puel: Sur une classe de problèmes non linéaires avec non linéarité positive, croissante, convexe. - Publications de I'UER de Math. de Lille 1, 1978.

[38] Montenegro, M.: Strong maximum principles for super-solutions of quasilinear elliptic equations. - Nonlinear Anal. 37, 1999, 431-448.

[39] Morabito, F.: Radial and non-radial solutions to an elliptic problem on annular domains in Riemannian manifolds with radial symmetry. - J. Differential Equations 258, 2015, 1461-1493.

[40] Nedev, G.: Regularity of the extremal solution of semilinear elliptic equations. - C. R. Acad. Sci. Paris Sér. I Math. 300, 2000, 997-1002.

[41] Sanchón, M.: Boundedness of the extremal solution for some $p$-Laplacian problems. - Nonlinear Anal. 67, 2007, 281-294.

[42] Tolksdorf, P.: Regularity for a more general class of quasilinear elliptic equations. - J. Differential Equations 51, 1984, 126-150.

[43] WeI, L.: Boundedness of the extremal solution for some $p$-Laplacian problems. - Math. Slovaca 64, 2014, 379-390.

[44] ZHANG, H.: Lower bounds for the first eigenvalue of the $p$-Laplace operator on compact manifolds with nonnegative Ricci curvature. - Adv. Geom. 7:1, 2007, 145-155. 